\title{
Hábitos alimentarios en universitarios que cursaron la materia de nutrición
}

\author{
Romo- López Diana Natalie*
}

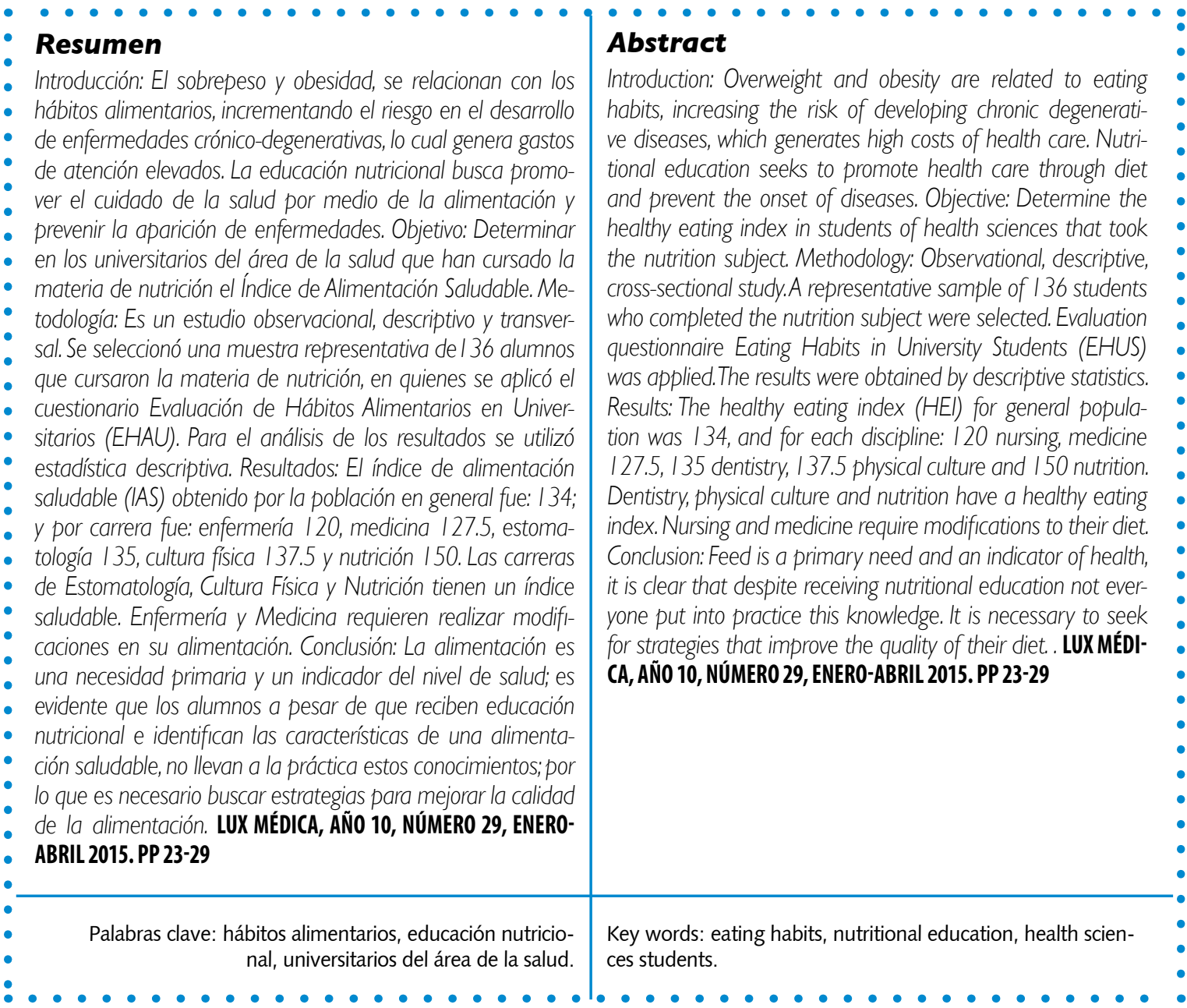

Profesora Investigadora de la carrera de Nutrición del Centro de Ciencias de la Salud de la Universidad Autónoma de Aguascalientes.

Fecha de recibido: 21 de enero 2015

Fecha de aceptado: 28 de febrero 2015

Correspondencia: LNC Diana Natalie Romo López. Departamento de Nutrición y cultura Física del Centro de Ciencias de la Salud de la Universidad Autónoma de Aguascalientes. Avenida Universidad Número 940. Ciudad Universitaria Código Postal 20131. Aguascalientes, Ags., México. Teléfono +52(449)9108443. Correo electrónico diana natalie@hotmail.com 


\section{Introducción}

El sobrepeso y la obesidad son un problema de salud pública en México, relacionado directamente con los hábitos alimentarios de la población. De acuerdo con la Encuesta de Salud y Nutrición 2012 (ENSANUT 2012) el 70\% de los adultos padece algún grado de sobrepeso u obesidad, lo cual es un factor de riesgo para el desarrollo de enfermedades crónico degenerativas; afectando el sistema económico y productivo del país. ${ }^{1,2}$ La Organización Mundial de la Salud (OMS) y la Organización Panamericana de la Salud (OPS) mencionan que los profesionales del área de la salud son los responsables de fomentar estilos de vida saludables en la población. ${ }^{3}$

La educación nutricional de acuerdo a Aranceta orienta sus recursos hacia el aprendizaje, adecuación y aceptación de hábitos alimentarios saludables con la cultura alimentaria, persiguiendo como objetivo un estado nutricio óptimo, disminuir la morbi-mortalidad de enfermedades relacionadas con una inadecuada alimentación. ${ }^{4}$ A pesar de que la alimentación es una necesidad primaria y un indicador del estado de salud, es evidente que hay una carencia en educación nutricional, lo cual se refleja en el estado nutricio de la población..$^{1,5}$

Un hábito alimentario de acuerdo a Bourgues se conceptualiza como un conjunto de conductas que se repiten y se conservan debido a la satisfacción que generan de manera consciente o no; en relación a la alimentación, entendiendo ésta como proceso que consiste en obtener del entorno alimentos, considerando la disponibilidad y aprendizaje de cada individuo y que está influido por factores socioeconómicos, psicológicos, culturales, religiosos y geográficos. ${ }^{6}$ Maffera $^{7}$ y Ledo-Varela ${ }^{8}$ refieren que la alimentación del universitario del área de la salud no es adecuada, teniendo un consumo de alimentos con abundantes azúcares simples, grasas saturadas, alimentos con alto aporte calórico, consumo elevado de sodio y hábitos alimentarios no adecuados como la omisión de comidas, entre otros.

La formación de hábitos se da generalmente durante las primeras etapas de la vida; sin embargo al ser una conducta, pueden modificarse de acuerdo al conocimiento que se adquiera en el desarrollo, por lo que la educación nutricional apoya a la generación de esas modificaciones, promoviendo hábitos alimentarios correctos. ${ }^{9}$

La educación alimentaria no es el único factor que influye en el aprendizaje de los hábitos alimentarios, si no también entran los factores sociales, culturales y económicos; es posible que el nivel de conocimiento en temas de nutrición y alimentación determine los hábitos alimentarios de manera individual. ${ }^{10} \mathrm{El}$ efecto de la educación nutricional, por medio de programas educativos sobre los hábitos alimentarios se han observado tanto resultados positivos como nulos. ${ }^{11-14}$

El ser humano de acuerdo a la teoría educativa del constructivismo se apropia del conocimiento por medio de la interacción y 
combinación día a día de aspectos cognitivos, sociales, de comportamiento, afectivos, ambiente y disposiciones internas. Por lo que se habrá generado un aprendizaje significativo en el momento en el que el alumno lo lleve a la práctica por medio del autocuidado y fomento a la salud. ${ }^{15-17}$ Suverza menciona que la evaluación de los hábitos alimentarios busca detectar riesgos de salud relacionados con la alimentación, de tal manera que se pueda facilitar una intervención oportuna. ${ }^{18}$ El objetivo de la investigación fue conocer el índice de alimentación saludable en los universitarios del área de la salud que han cursado la materia de nutrición.

\section{| | | | | | | | | | | | | | | | | | | | | | | | | | | | | | | | | | | | | | | | | | | | | | | | | | | | | | | | | | | | | | | | | | | | | | | | | | | | | | | | | | | | | | | | | | | | | | | | | | | | | | ||}

\section{Material y métodos}

Se realizó un estudio observacional, descriptivo y transversal. El universo, estuvo conformado por 207 alumnos del área de la salud que cursaron la materia de nutrición, en el semestre enero-junio 2014. Se obtuvo una muestra representativa de 136 alumnos, con un margen de error de $5 \%$ y un nivel de confianza del 95\%. Se desarrolló el cuestionario Evaluación de Hábitos Alimentarios en Universitarios (EHAU) en modalidad electrónica en línea, que estuvo conformado por enunciados relacionados con la frecuencia de consumo de alimen- tos, los cuales se respondieron de acuerdo a una escala de Likert.

La asignación de puntaje a cada enunciado sirvió para determinar el Índice de Alimentación Saludable en Universitarios (IASU). La clasificación del IASU se realizó de acuerdo a la puntuación total y se dividió en tres categorías, quedando de la siguiente manera: 0-67 poco saludable (hábitos alimentarios malos), 68-134 necesita cambios (hábitos alimentarios regulares) y de 135-200 como saludable (hábitos alimentarios saludables). Para el análisis de datos se utilizó estadística descriptiva.

\section{| | | | | | | | | | | | | | | | | | | | | | | | | | | | | | | | | | | | | | | | | | | | | | | | | | | | | | | | | | | | | | | | | | | | | | | | | | | | | | | | | | | | | | | | | | | | | | | | | | | | ||}

\section{Resultados}

La edad promedio de los 136 universitarios incluídos fue de $20 \pm 1.9$ años. La distribución de participación por género fue 59\% femenino y $41 \%$ masculino. La colaboración por carrera fue Cultura Física con un $18.5 \%$, Enfermería con un $22.3 \%$, Estomatología con un 23.7\%, Nutrición con un $18.5 \%$ y Medicina con un $17 \%$.

Al evaluar los hábitos alimentarios encontramos que el $28 \%$ de los universitarios que cursaron la materia no desayunan antes de iniciar sus actividades académicas o laborales y el $72 \%$ sí lo realiza. Las razones por las que no desayunan fueron $74 \%$ por falta de tiempo, $18 \%$ porque no acostumbra y $3 \%$ por falta apetito. De acuerdo al número de comidas el $71 \%$ realiza entre $3(34 \%)$ y $4(37 \%)$ tiempos de comidas, seguido con $14 \%$ quienes consumen 5 tiempos de comida, el $11 \%$ realiza 2 comidas y únicamente el $4 \%$ hace más de cinco comidas. El momento en que se consumen mayor cantidad de alimentos en el día es la comida con $58 \%$, seguido del desayuno con $21 \%$, el $11 \%$ destina mayor cantidad de comida al almuerzo y el $10 \%$ a la cena.

En la figura 1 se agruparon los alimentos que se deben consumir para tener una alimentación saludable, el consumo de frutas en promedio fue de 2 a 5 veces por semana, el consumo de cereales integrales de 2 a 5 veces por semana, el de grasas con un 
perfil adecuado una vez a la semana, el de carnes con poca grasa de 2 a 5 veces por

semana y el de leguminosas de 2 a 5 veces por semana.

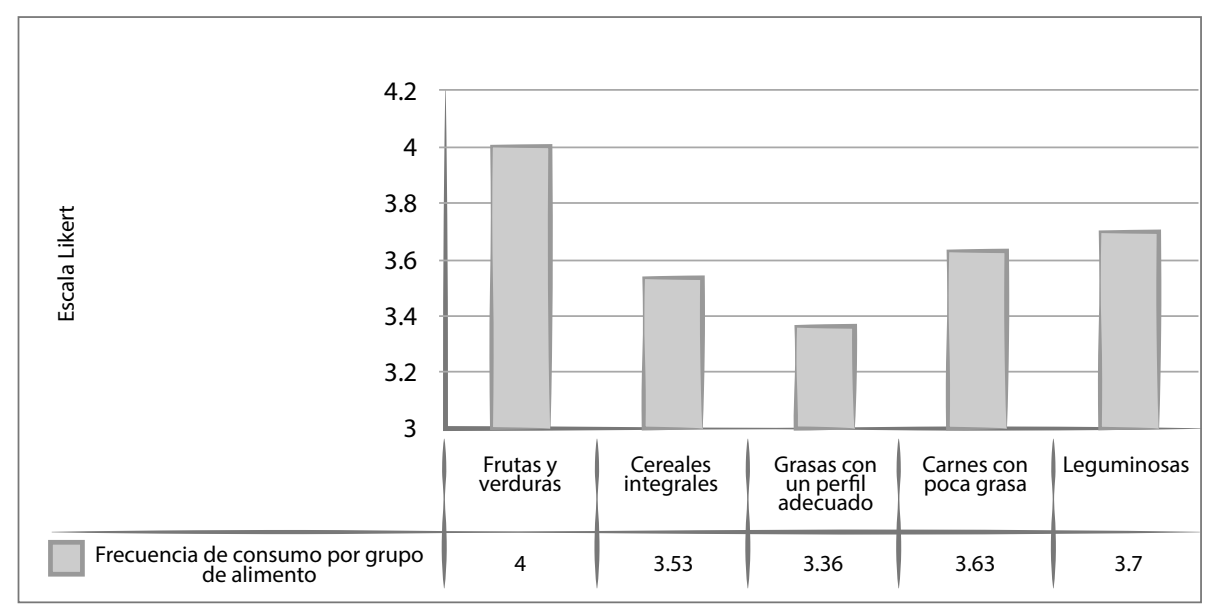

$n=136$ alumnos

Escala Likert: 1-Nunca 2-Una vez al mes 3-Una vez a la semana 4-Entre 2 y 5 veces a la semana 5-Diario

Figura 1. Hábitos alimentarios. Frecuencia de consumo por grupo de alimentos con nutrimentos saludables

En la figura 2 se agruparon los alimentos que contienen nutrimentos no recomendados, debido a su efecto dañino en el organismo de las personas que los consumen, de acuerdo a lo mencionado en el cuestionario aplicado, el promedio de consumo de los alimentos con alto contenido calórico se consumen de 2 a 5 veces por semana, las botanas se consumen una vez a la semana, los alimentos con alto contenido de grasas saturadas una vez a la semana, los refrescos y jugos industrializados también una vez a la semana y los alimentos de alto contenido en sodio una vez al mes.

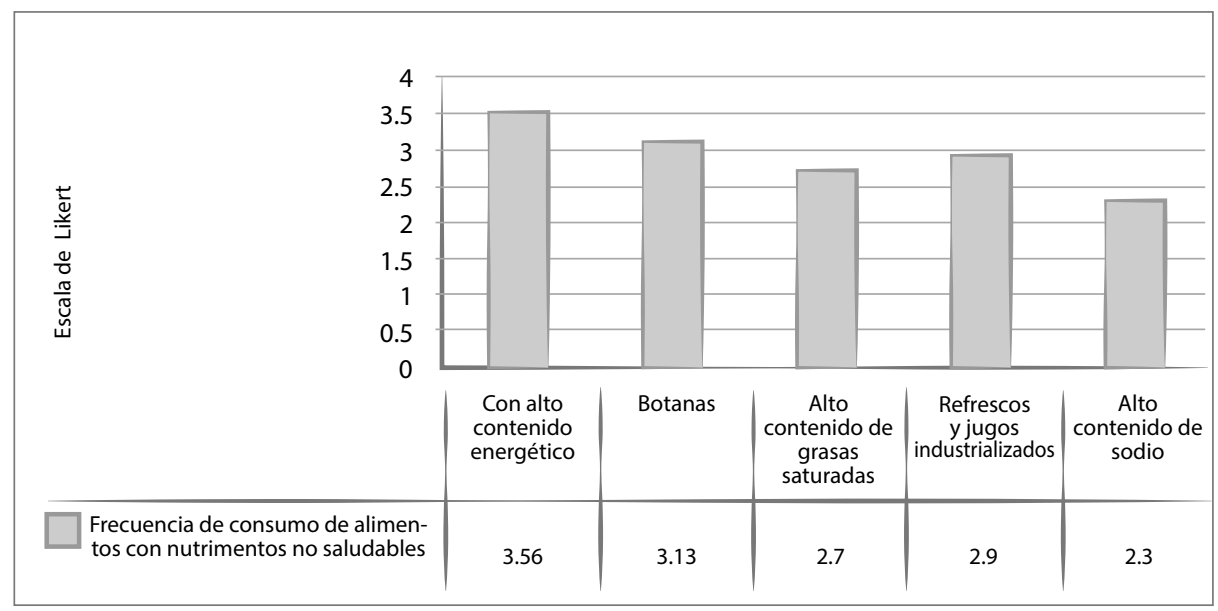

$n=136$ alumnos

\section{Escala Likert: 1-Nunca 2-Una vez al mes 3-Una vez a la semana 4-Entre 2 y 5 veces a la semana 5-Diario}

Figura 2. Hábitos alimentarios. Frecuencia de consumo por grupo de alimentos con nutrimentos no saludables. 
Con los datos recolectados se determinó el IASU de la población general y de cada una de las carreras que incluyen la materia de nutrición. En la figura 3 se muestra el puntaje obtenido en el IASU. La población general requiere realiza modificaciones en su alimentación. Estomatología, cultura física y nutrición tienen un índice de alimentación saludable. Enfermería y medicina requieren realizar modificaciones en su alimentación.

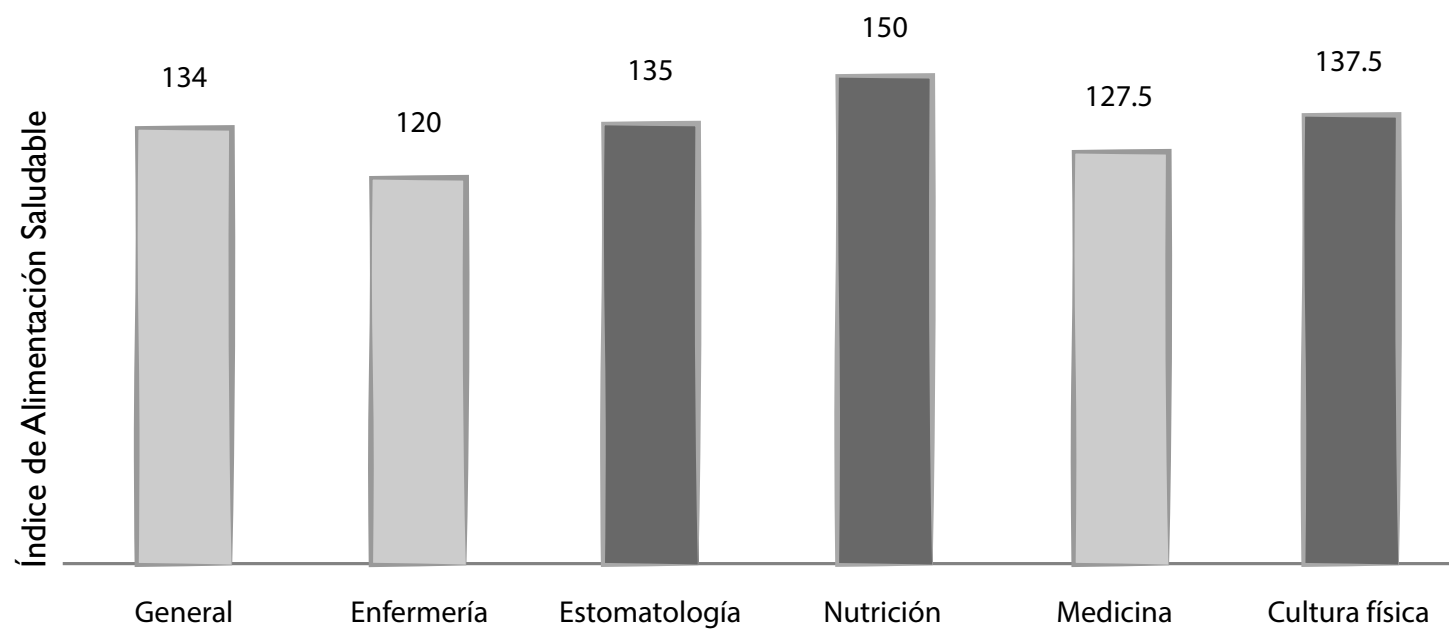

$\mathrm{n}=136$ alumnos

Interpretación IASU: 0-67 poco saludable (hábitos alimentarios malos), 68-134 necesita cambios (hábitos alimentarios regulares) y de 135-200 como saludable (hábitos alimentarios saludables)

Figura 3. Hábitos alimentarios. Índice de Alimentación Saludable en Universitarios.

\section{Discusión}

Gómez y Salazar encontraron en la Universidad Veracruzana que las facultades de Enfermería, Medicina y Odontología perciben sus hábitos alimentarios como regulares; ${ }^{19}$ dato similar al encontrado ya que el $59 \%$ considera que tiene hábitos regulares, el $25 \%$ los considera buenos, el $14 \%$ los considera malos y el $2 \%$ los considera muy buenos.

Generalmente el universitario es consciente de que las características de su alimentación no son del todo correctas y como lo describen algunos expertos en donde se menciona que es muy común que salte comidas, tenga periodos de ayuno prolongados, realice comidas con prisa e incluso realicen actividades al mismo tiempo, como comer y estudiar; lo preocupante es que el universitario justifica esas conductas debido a que sus horarios de estudio complican sus tiempos de comida y que posiblemente una vez que terminen con sus estudios no volverán a realizar este tipo de conductas alimentarias, sin embargo como bien lo mencionó Wardle un hábito alimentario requiere de 66 días para que se convierta en una acción automatizada, el universitario en promedio estaría repitiendo estas conductas al menos 4 años, si es un alumno regular, por lo que muy probablemente muchas de las conductas relacionadas con su alimentación formen hábitos alimentarios perdurables. ${ }^{11,12,19,20}$ 
El desayuno de acuerdo a Servín en Téllez 2010, es el tiempo de comida más importante, ya que provee los nutrimentos y la energía necesaria para iniciar y realizar las actividades físicas e intelectuales. En esta investigación los universitarios que desayunan antes de iniciar actividades son el $72 \%$ y el $28 \%$ no lo hace, dato muy similar al encontrado por Saad en 2007 donde el $75 \%$ sí desayuna y el $25 \%$ no lo hace. La formación de este hábito generalmente es en la infancia, sin embargo el acceso a la universidad es un momento crucial en el que se pueden modificar los hábitos alimentarios debido a los horarios, el nivel de estrés y carga a la que están sometidos; pero de repetir estas conductas erróneas posiblemente se establezcan de por vida, llevándolos a desarrollar enfermedades como el sobrepeso, la obesidad y enfermedades crónico degenerativas no trasmisibles. ${ }^{21,22}$
El resultado obtenido en el IASU es comparable al encontrado en la Universidad de Castilla de España, por Cervera en 2013 donde el 80\% de la población universitaria se encontraba en una situación en la que era necesario realizar cambios en su forma de alimentarse, sin embargo la proporción de estudiantes que requieren un cambio es menor ya que únicamente dos (Enfermería y Medicina) de las cinco carreras obtuvieron dicho resultado en que fuera necesario realizar cambios en su forma de alimentarse. ${ }^{23}$

Sí bien la educación nutricional influye, no es el único factor que determina la manera de alimentarse de las personas; posiblemente el nivel de conocimiento pueda o no tener influencia, en esta investigación se pudo observar que los universitarios de la carrera de nutrición tienen mejores hábitos, sin embargo es claro que es su área de especialización, y por lo tanto la motivación también varíe de una carrera a otra.

\section{Conclusión}

La generación de aprendizajes significativos no se presenta en los alumnos que cursan la materia de nutrición, ya que no hay un autocuidado en su alimentación; las posibles causas de este resultado son como que la alimentación es un proceso complejo en el que intervienen infinidad de factores como geográficos, psicológicos, culturales, religiosos, la disponibilidad, el aspecto afectivo, los gustos y creencias, entre otros, que afectan las decisiones que realiza el ser humano al momento de seleccionar alimentos.De acuerdo a nuestros resultados, es conveniente que se apoye a los estudiantes para que modifique su alimentación hacia una saludable.

\section{Bibliografía}

1. Encuesta Nacional de Salud y Nutrición 2012 [Internet] (Consultado 2014 Junio 15) Disponible en: http://ensanut.insp.mx/informes/ENSANUT2012ResultadosNacionales.pdf

2. Casanueva, E. Kaufer, M. Pérez, A. y Arroyo, P. Nutriología médica. 3ra edición. México. Editorial medica panamericana. Pág. 822. 2008
3. Organización Mundial de la Salud. Capítulo 38: Fomento de dietas y estilos de vida saludables. [Internet] (Consultado 2014 Marzo 16) Disponible en: http://www.fao.org/docrep/006/w0073s/ w0073s16.htm\#TopOfPage

4. Aranceta, Javier. Nutrición comunitaria. 2nda edición. España. Editorial Masson. Pág. 284. 2001

5. Brown, Judith E. Nutrición en las diferentes etapas de la vida. 5ta edición. Stamford. Mc-Graw Hill. Pág. 408. 2014 
6. Bourgues, Héctor. Costumbres, prácticas y hábitos alimentarios. Cuadernos de nutrición. 1990; 13:1732

7. Maffera, Julieta. ¿Cómo se alimentan los estudiantes universitarios? Edición uncuyo la publicación digital de la universidad. 6 Mayo 2011 [Internet] (Consultado 2014 Marzo 1) Disponible en: http://www.edicionuncuyo.com/dieta-universitaria

8. Ledo-Varela, Ma. T. De Luis Román, D. A. GonzálezSagrado, M. Izaola Jauregui, O. Conde Vicente, R. Aller de la Fuente, R. Características nutricionales y estilo de vida en universitarios. Nutrición Hospitalaria. 2011; 26: 814-818

9. Cervera, P. Clapés, J. Rigolfas, R. Alimentación y dietoterapia. 4ta edición España. McGraw-Hill Interamericana. pág. 280. 2004

10. Contreras, P. Camacho, E. Ibarra, M. Ramón, R. Escoto, M. Pereira, C y Munguía, L. Hábitos alimentarios en estudiantes universitarios. Revista digital universitaria. 2013; 14:1-15

11. Vidal, M; Díaz, A; Jiménez, C y Quejigo, J. Modificación de ciertas actitudes frente a la alimentación en estudiantes de enfermería tras cursar la asignatura de nutrición y dietética. Revista de Nutrición Clínica y Dietética Hospitalaria. 2012; 32: 48-58

12. Rivera, María. Hábitos alimenticios en estudiantes de la Universidad Juárez Autónoma de Tabasco. Revista Cubana de Salud Pública. 2006; 32: 0-0

13. Romero Hernández, Edith. Promoción de estilos de vida saludables en estudiantes de la facultad de Nutrición Xalapa de la Universidad Veracruzana. [Tesis de pregrado] Universidad Veracruzana; 2013

14. Montero, A. Úbeda, N. y García, A. Evaluación de los hábitos alimentarios de una población de estudiantes universitarios en relación con sus conocimientos nutricionales. Revista nutrición hospitalaria. 2006; 21:466-473
15. Carranza, Alba Irene. El constructivismo como estrategia educativa: formación profesional en enfermería. Enfermería en Costa Rica. 2005; 15-22

16. Díaz Barriga, Frida. Hernández Rojas, Gerardo. Estrategias docentes para un aprendizaje significativo: una interpretación constructivista. 1ra. Edición. México. Mc-Graw Hill. Pág. 405. 2010

17. Mecalco Herrera, Cecilia. Formación de estudiantes universitarios como promotores de salud, bajo un enfoque constructivista. [Internet] (Consultado 2014 Junio 15) Disponible en: http://www.paho.org/saludyuniversidades/index.php?option $=$ com_content \&view $=$ article\&id $=37 \&$ Itemid $=59 \&$ lang $=$ es

18. Suverza, A y Haua, K. El abcd de la evaluación del estado de nutrición. 1era. edición. México. Mc-Graw Hill. pp. 332. 2010

19. Gómez, J y Salazar, N. Hábitos alimenticios en universitarios de Ciencias de la Salud de Minatitlán, Veracruz. [Tesis de pregrado] pp. 39. 2010

20. Lally, P. Jaarsveld, C. y Walden, J. How are habits formed: modeling habit formation in the real world? European Journal of Social Psychology. 2010;40: 998-1009

21. Servín Rodas, María del Carmen. Téllez Villagómez, $M$. Nutrición en las diferentes etapas de la vida. 1era. edición. México. Manual Moderno pp.332.

22. Saad, c. Ibáñez, E. León, C. Colmenares, C. Vega, N y Díaz, Y. Cambios en los hábitos alimentarios de los estudiantes de enfermería de la Universidad El Bosque, durante su proceso de formación académica. Revista Colombiana de Enfermería. 2007; 3: 51-60

23. Cervera, F. Serrano, R. Vico, C. Milla, M. García, M. Hábitos alimentarios y evaluación del estado nutricional en una población universitaria. Revista Nutrición Hospitalaria. 2013; 28: 438-446 